\title{
Recurrencia de crisis febriles en una población chilena
}

\author{
MARÍA ESTER PIZARRO ${ }^{1}$, HERNÁN BORJAA, CAROLINA CORIA DE LA H. ${ }^{2}$, \\ LUIS VILLARROEL DEL P. ${ }^{3}$, JAIME CERDA L. ${ }^{3}$, TOMÁS MESA L. ${ }^{4}$, \\ TAMARA HIRSCH B. ${ }^{1 \mathrm{~A}}$, RAÚl ESCOBAR H. ${ }^{4}$, MARTA HERNÁNDEZ $\mathrm{CH}^{4}{ }^{4}$ \\ 1. Departamento de Pediatría Facultad de Medicina Pontificia Universidad Católica de Chile, (1A) Servicio de urgencia Infantil. \\ 2. Neurología Infantil Hospital Herminda Martín de Chillán. \\ 3. Departamento de Salud Pública Facultad de Medicina Pontificia Universidad Católica de Chile. \\ 4. Sección Neurología Infantil Pontificia Universidad Católica de Chile, Santiago. \\ A. Interno Escuela de Medicina Facultad de Medicina, Pontificia Universidad Católica de Chile.
}

\begin{abstract}
Febrile seizures recurrence in Chilean children

Objective: Evaluate the clinical presentation and risk factors for recurrence of first febrile seizure (FS) in patients at Pediatric Emergency Department or Neurology Units at Clinical Hospital Universidad Católica (UC) and Hospital Herminda Martin from Chillán (HM). Method: Descriptive-observational study of children with age average of 2.9 years-old, with first febrile seizure between January 1, 2003 and December 31, 2005. Results: Total 158 patients; $76 \%$ presented a simple FS and $24 \%$ a complex FS as first episode, only 1 case with febrile epileptic status. In $22 \%$ and $18 \%$ respectively, there was a FS history and epilepsy in first degree relatives. $34 \%$ of patients presented a second episode; $36 \%$ of these cases present a third FS and $9 \%$ more than 3 episodes. The average time of recurrence was 6.9 months. The most important risk factors for recurrence were: epilepsy history in first degree relatives and type of febrile seizure, with hazard ratio of $2.5(\mathrm{p}=0.001)$ and $1,8(\mathrm{p}=0.03)$ respectively. When fitting both variables, only family history of epilepsy was significant. Conclusions: Most of FS episodes are simple and 34\% present recurrence. Family history of epilepsy and type of febrile seizure are associated recurrence risk factors. The follow-up of these patients does not predict their future risk of epilepsy.

(Key words: febrile seizures, recurrence, epilepsy).

Rev Chil Pediatr 2008; 79 (5): 488-494
\end{abstract}

\section{RESUMEN}

Objetivo: Evaluar la presentación clínica, recurrencia y factores de riesgo de recurrencia en un grupo de pacientes con primoconvulsión febril que consultaron en Urgencia pediátrica y/o consulta neurológica en los hospitales Clínico Universidad Católica (UC) y Herminda Martín de Chillán (HM). Método: Estudio

Trabajo recibido el 20 de marzo de 2008, devuelto para corregir el 07 de julio de 2008, segunda versión el 09 de julio de 2008, aceptado para publicación el 16 de julio de 2008.

Correspondencia a:

Dra. Marta Hernández Ch.

E-mail: mhernand@med.puc.cl 
observacional descriptivo de niños con primoconvulsión febril entre el $1^{\circ}$ de Enero del 2003 y el $31^{\circ}$ de Diciembre 2005 con seguimiento promedio de 2,92 años. Resultados: De 158 pacientes, el 76\% debutó con una CF simple y el $24 \%$ con una CF compleja (1 status febril). En el 22 y $18 \%$ había antecedentes de CF y epilepsia en familiares de primer grado respectivamente. Recurrieron 34\% de los pacientes y de ellos el 36\% presento una tercera CF y el 9\% más de tres episodios. El promedio de tiempo de recurrencia fue 6,9 meses. Los factores de riesgo más importantes de recurrencia fueron el antecedente de epilepsia en familiares de primer grado y tipo de crisis con un hazard ratio de $2,5(\mathrm{p}=0,001)$ y $1,8(\mathrm{p}=0,03)$ respectivamente. Al ajustar ambas variables sólo antecedentes familiares de epilepsia fue significativa. Conclusiones: La mayoría de las CF es simple y recurre un 34\%. Los antecedentes familiares de epilepsia y tipo de crisis son factores de riesgo asociados a recurrencia). El seguimiento no permite evaluar el riesgo de epilepsia a futuro en estos pacientes.

(Palabras clave: crisis febriles, recurrencias, epilepsia).

Rev Chil Pediatr 2008; 79 (5): 488-494

\section{Introducción}

La crisis febril (CF) es el evento convulsivo más frecuente en pediatría, se presenta entre los tres meses y cinco años asociados a fiebre, sin evidencia de infección intracraneal, en niños sin patología neurológica ni crisis afebriles previas $^{1}$. Su mayor frecuencia aparece entre los 18 y 22 meses. Se describe una incidencia de 2 a $5 \%{ }^{2}$ en Europa y Norteamérica, y $9-10 \%$ en Japón ${ }^{3}$.

A pesar que la mayoría de las crisis febriles tienen una duración de uno a dos minutos, provocan pánico en los padres, quienes relatan el evento con el sentimiento de amenaza vital y se preguntan la posibilidad de recurrencia, riesgo de epilepsia, daño neurológico secundario o muerte durante el evento.

Hay extensos estudios en la literatura internacional que describen la historia natural de las CF. Datos de 5 grandes cohortes muestran que recurren un tercio de los pacientes y el riesgo de desarrollar epilepsia después de una primoconvulsión febril es un 1-2\%, levemente superior al de la población general ${ }^{4,5}$. En ninguna se ha descrito daño neurológico secundario, aún cuando la presentación fue un status febril ${ }^{6,7} \mathrm{y}$ la mortalidad, que teóricamente pudiera ocurrir, no se ha reportado aún ${ }^{8}$.

No existen estudios locales recientes (en la última década) que informen sobre recurrencia, factores de riesgo asociados y evolución; nuestras respuestas actuales se basan en datos de literatura extranjera. Nos enfrentamos a la ne- cesidad de tener un estudio nacional que aporte datos de nuestra población.

La hipótesis en la que se proyectó este trabajo es que la recurrencia de CF es similar a lo descrito en la literatura internacional. Se analizaron como factores de riesgo para recurrencia edad de la primera crisis, antecedentes en familiares de primer grado de crisis febriles y epilepsia, tipo de crisis, tiempo entre la crisis y el inicio de la fiebre

\section{Pacientes y Métodos}

Estudio observacional, descriptivo de un grupo de niños que consultaron por primoconvulsión febril en Urgencia pediátrica o consulta neurológica en los hospitales Clínico Universidad Católica (UC) y Herminda Martín de Chillán (HM) desde el $1^{\circ}$ de Enero del 2003 y el $31^{\circ}$ de Diciembre 2005.

Pacientes: Se revisaron las fichas clínicas de urgencia pediátrica y policlínicos ambulatorios en la Red de Salud UC y en el Hospital Herminda Martín de Chillán entre el $1^{\circ}$ de Enero del 2003 y el $31^{\circ}$ de Diciembre 2005 y se detectaron los pacientes que habían tenido una primoconvulsión. Identificadas las fichas clínicas se les solicitó a los médicos tratantes (pediatra o neurólogo infantil) investigar anammésticamente factores de riesgo de recurrencia, evaluar clínicamente el desarrollo psicomotor, desde el punto de vista motor y cognitivo, las recurrencias de evaluación y el tratamiento, 
para lo cual se les envió una ficha electrónica estandarizada.

A los pacientes que no continuaron en control, se les realizó una entrevista telefónica personal por parte de uno de los neurólogos, evaluando recurrencias, desarrollo psicomotor y los demás aspectos contenidos en la ficha electrónica. Se solicitó consentimiento informado a los padres de los pacientes.

Los criterios de inclusión fueron primera $\mathrm{CF}$ en pacientes con desarrollo psicomotor normal, sin infección meníngea intercurrente. Se excluyeron los pacientes con crisis febriles o afebriles previas o daño neurológico previos. Los factores de riesgo consultados fueron: antecedentes familiares de primer grado de crisis febriles y epilepsia, edad de primera crisis, tipo de crisis, tiempo entre el inicio de la fiebre y la $\mathrm{CF}$, tratamiento en servicio de urgencia y al alta. Se definió la CF simple como única, generalizada y con duración menor de 15 minutos; y CF compleja cuando era múltiple, focal y mayor de 15 minutos 9 .

Para el análisis estadístico se estimó el tiempo libre de recurrencia con el método de KaplanMeier, se compararon las curvas de sobrevida según diferentes factores de riesgo con el test de Log-rango. Se ajustó un modelo de riesgos proporcionales de Cox para identificar factores asociados en forma conjunta para riesgos de recurrencia y dimensionar la cuantía de dicho riesgo. Se fijó un límite de significancia estadística alfa menor o igual a 0,05 y que se utilizó el paquete estadístico SPSS.

\section{Resultados}

Se identificaron 192 pacientes y se realizó seguimiento completo a $158(83 \%)$. Durante el seguimiento hubo una pérdida de 34 pacientes $(17 \%)$ de la muestra.

Del total de pacientes incluidos 99 (63\%) correspondían a género masculino, la edad de presentación del primer episodio fue de 18 meses como mediana con un rango entre 4-60 meses (figura 1). La primoconvulsión fue simple en $120(76 \%)$ y compleja en 38 (24\%), un paciente debutó con un status epilépticus febril. Durante el evento agudo 70 (44\%) pacientes se hospitalizaron y a $43(28 \%)$ se le realizó una punción lumbar.

Tenían antecedentes familiares de primer grado de crisis febril 33 (21\%) y de epilepsia 28 (18\%). En 125 (81\%) la crisis febril se presentó en las primeras 24 horas del cuadro febril, en 21 $(13 \%)$ se presentó después de 24 horas y en 8 (6\%) la crisis febril correspondió al primer síntoma de enfermedad. Presentaron la primera $\mathrm{CF}$ antes de los 18 meses, 90 niños (57\%). Se realizó electroencefalograma (EEG) a 75 pacientes $(47 \%)$, de estos 61 resultaron normales $(81 \%)$ y $14(19 \%)$ resultaron alterados mos-

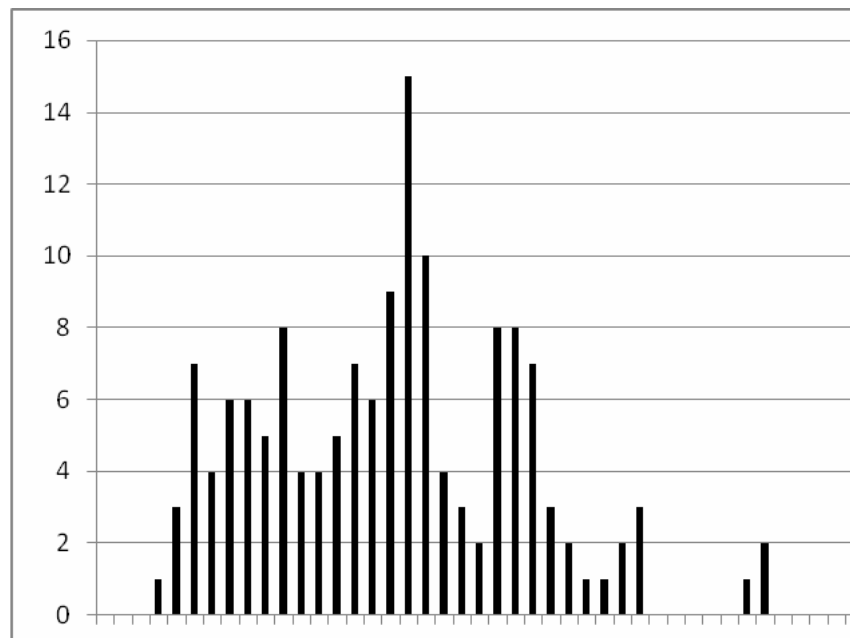

13579111315171921232527293133353739414
Figura 1. Distribución etaria de la primera crisis febril en la cohorte estudiada. 
trando actividad irritativa epileptiforme. Se realizó neuroimagen (Tomografía axial computada o Resonancia nuclear magnética) a 36 pacientes $(23 \%)$, de estos resultaron normales 29 (80\%) y anormales 7 (20\%). Las anomalías correspondieron a híper intensidades de sustancia blanca peri ventricular inespecíficas.

Posterior al primer episodio, a 140 pacientes se les indicó tratamiento para prevención de crisis prolongadas o status febril en forma intermitente con diazepam $0,5 \mathrm{mg} / \mathrm{kg}$ vía rectal en caso de crisis y a 14 pacientes $(9 \%)$ se le indicó tratamiento profiláctico continuo de crisis febriles con fenobarbital o ácido valproico. De estos pacientes 10 presentaron recurrencia.

El seguimiento fue entre 2 y 4,8 años con una mediana de 2,9 años. Cuatro niños evolucionaron con epilepsia y uno de ellos con un síndrome de Dravet los que se excluyeron del análisis posterior quedando 154 pacientes. De los pacientes incluidos ninguno evolucionó con retraso del desarrollo psicomotor.

El 34,4\% de los niños presentaron un segundo episodio de CF, y de estos un 36\% y $9 \%$ presentaron un tercer y más de tres episodios

Función de sobrevida según antecedentes familiares de epilepsia

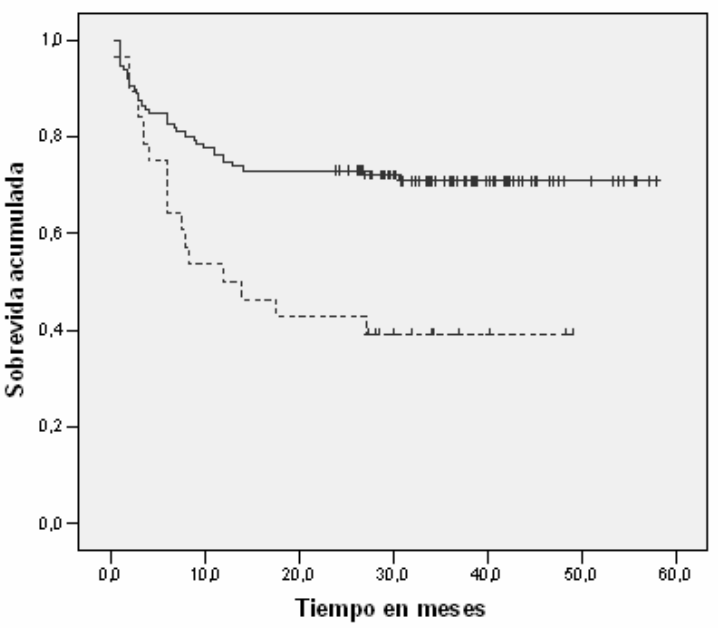

Posteriormente, se comparó la probabilidad de recurrencia según la presencia de diferentes factores de riesgo mediante el test de logrango. Los factores de riesgo fueron: antecedentes familiares de epilepsia, antecedentes familiares de crisis febril, edad de primera crisis, tipo de crisis y tiempo entre inicio de la fiebre y aparición del evento (tabla 1). Los pacientes con antecedentes de epilepsia en familiares de primer grado presentaron una mayor probabilidad de recurrencia, al igual que los pacientes con crisis complejas ( $\mathrm{p}=0,03$, figura 2$)$.

Tabla 1. Comparación de distintos factores de riesgo para crisis febriles

\begin{tabular}{|c|c|c|c|}
\hline Factores de Riesgo & n de pacientes & $(\%)$ & Valor p \\
\hline $\begin{array}{l}\text { Antecedentes familiares } \\
\text { de epilepsia }\end{array}$ & $\begin{array}{l}\text { Si: } 28 \\
\text { No: } 126\end{array}$ & $\begin{array}{l}(18 \%) \\
(82 \%)\end{array}$ & $0,001+$ \\
\hline $\begin{array}{l}\text { Antecedentes familiares } \\
\text { de convulsiones febriles }\end{array}$ & $\begin{array}{l}\text { Si: } 33 \\
\text { No: } 121\end{array}$ & $\begin{array}{l}(21 \%) \\
(79 \%)\end{array}$ & ns \\
\hline Tipo de crisis & $\begin{array}{l}\text { Simple: } 117 \\
\text { Compleja: } 37\end{array}$ & $\begin{array}{l}(76 \%) \\
(24 \%)\end{array}$ & $0,03+$ \\
\hline $\begin{array}{l}\text { Duración de la fiebre } \\
\text { previo a la crisis }\end{array}$ & $\begin{array}{l}>24 \text { horas: } 21 \\
<24 \text { horas: } 133\end{array}$ & $\begin{array}{l}(13 \%) \\
(87 \%)\end{array}$ & ns \\
\hline $\begin{array}{l}\text { Edad de presentación } \\
1^{\circ} \text { crisis }\end{array}$ & $\begin{array}{l}=18 \text { meses: } 90 \\
>18 \text { meses: } 64\end{array}$ & $\begin{array}{l}(58 \%) \\
(42 \%)\end{array}$ & ns \\
\hline
\end{tabular}

Figura 2. Curva de Kaplan Meier que compara sobrevida libre de recurrencia en pacientes con antecedentes familiares de primer grado de epilepsia y tipo de crisis febril compleja, (línea punteada) versus pacientes sin antecedentes familiares y crisis febril simple (línea continua). 
Se ajustó un modelo de riesgos proporcionales de Cox incluyendo en forma univariada la variable "antecedentes familiares de epilepsia", la cual presentó un riesgo de 2507 veces más recurrencia (IC95\% 1 406-4 472; p = 0,002) para pacientes con antecedentes familiares de epilepsia versus pacientes sin este antecedente. Se realizó el mismo análisis para la variable "tipo de crisis", obteniéndose un riesgo de 1,86 veces más (IC95\% 1 053-3 288; p = 0,033) para los pacientes que presentaron crisis complejas.

Finalmente, ambas variables se introdujeron al modelo de riesgos proporcionales de Cox, con el fin de ajustar una por la otra y viceversa. Solamente la variable antecedentes familiares de epilepsia fue estadísticamente significativa, siendo su riesgo de 2305 veces (IC95\% 1,28$4,15 ; \mathrm{p}=0,005)$.

\section{Discusión}

La visión de crisis febriles difiere mucho desde la mirada de los padres y médicos. Para los primeros es un evento que pone en riesgo la vida de su hijo y solicitan hospitalización y un estudio completo, mientras que para los segundos corresponde a un episodio benigno, donde una vez excluida la meningitis no debiera haber necesidad de realizar más exámenes a excepción del estudio y tratamiento del foco infeccioso. En relación a estas dos visiones contrapuestas, se observa que paradojalmente en un hospital se hospitaliza el $44 \%$ de los pacientes en su primer episodio y en el otro no hay hospitalizaciones por estos eventos. La mayoría de las veces la angustia parental es la que influye en esta decisión.

A través de los años se han producido varios cambios en el enfrentamiento a las CF. Entre 1930-1950 se veían como una condición benigna que no requería tratamiento ${ }^{9}$, en la década del 1960-1980, la esclerosis hipocampal secundaria atribuida a la CF y su relación a epilepsia del lóbulo temporal llevó a una explosión mundial de tratamiento con fenobarbital o acido valproico a largo plazo en miles de lactantes y preescolares ${ }^{9}$. Afortunadamente esta visión, gracias al estudio de cohortes, ha cambiado y nuevamente se vuelve a definir la
CF como una condición benigna, en la cual la intervención más importante es conversar con los padres y tranquilizarlos respecto a este evento ${ }^{10}$.

Las recomendaciones de la Guía Clínica para el manejo a largo plazo de las crisis febriles simples de la Academia Americana de Pediatría año $2008^{11}$ describe las principales interrogantes a responder a los padres ante una primoconvulsión a) ¿Hay una "secuela neurológica" secundaria a las crisis febriles? b) ¿hay riesgo de muerte durante la crisis? c) ¿cual es el riesgo de recurrencia? d) ¿cual es el riesgo de epilepsia?. Respecto a la primera pregunta, no hay literatura que describa declinación intelectual secundaria a las crisis febriles, Nuestro trabajo no evaluó en forma sistemática el desarrollo psicomotor, pero los pacientes con crisis febril simple mantuvieron un desarrollo acorde a los esperado según la información del pediatra o neurólogo tratante, pero es necesario realizar estudios prospectivos a largo plazo y con mediciones estandarizadas de desarrollo para contestar esta pregunta en forma más precisa. Respecto al desenlace fatal secundario a una crisis febril, a pesar que hay un riesgo teórico que un niño pueda fallecer durante una crisis secundario a una aspiración, arritmia cardíaca ${ }^{8}$, esta nunca ha sido reportada y en nuestra serie no fue observada.

Las recurrencias de las crisis febriles varían desde un 25-35\% según estudios internaciona1 es ${ }^{12}$, el $34 \%$ nuestra serie está cercana al límite. Los factores de riesgo de recurrencias descritos en grandes cohortes internacionales ${ }^{13}$ y considerados como "definitivos" ( sión) son historia familiar de crisis febriles, primoconvulsión en menor de 18 meses, grado de fiebre y duración de la fiebre. El antecedente familiar de epilepsia fue considerado un factor "posible" de riesgo y el tipo de crisis no fue considerado un factor de riesgo. Esto difiere con nuestros resultados, hecho que posiblemente se deba a la complejidad en recabar dicho antecedente en familiares de segundo grado en esas cohortes, especialmente considerando lo heterogénea que es la definición de crisis convulsiva. Nuestro trabajo sólo consideró a familiares de primer grado (padre, madre y hermanos). 
Con respecto al riesgo de epilepsia después de una primera crisis febril, éste no se pudo estimar por el tiempo de seguimiento realizado a nuestros pacientes, para lo cual necesitamos al menos 10 años, pero en el seguimiento 4/158 $(2,5 \%)$ pacientes tuvieron crisis afebriles.

La presentación clínica según tipo de crisis no difiere de la descrita, la más frecuente con un $76 \%$ fueron las crisis febriles simples, mayoritariamente en hombres $(63 \%)$ con una edad promedio de debut de 18 meses.

Uno de los resultados destacados fue un 9\% (14 pacientes) tratados en forma permanente después de una primoconvulsión, correspondiendo todos ellos a pacientes con crisis febril simple o compleja asociado descargas epileptiformes en el EEG. De estos niños tratados 10 presentaron recurrencia de $\mathrm{CF}$ y uno de ellos evolucionó con un síndrome de Dravet o epilepsia mioclónica severa de la infancia ${ }^{14}$. La AAP en su guía de 1996, establece que no existe evidencia que un EEG anormal después de una primoconvulsión sea predictivo de recurrencia o riesgo de epilepsia. En contraste la guía propuesta en Japón ${ }^{15}$ da especial énfasis al EEG y proponen el concepto de "crisis febril epiléptica" cuando el EEG revela descargas epileptiformes aun cuando la manifestación clínica es consistente con una crisis febril simple y recomiendan terapia anticonvulsivante diaria. Hay estudios que demuestran que el tratamiento diario no reduce el riesgo de recurrencia ni epilepsia ${ }^{16}$, como también lo sugiere nuestro trabajo.

En conclusión, apoyamos la idea de que las crisis febriles son eventos benignos, que no causan mortalidad ni morbilidad neurológica asociada y que la prevención debe ir dirigida a evitar una crisis prolongada más que una recurrencia.

Asimismo, los resultados de este estudio sugieren la indagación en mayor profundidad tanto de los antecedentes familiares de epilepsia y la definición del tipo de CF, pues ambos factores de riesgo están significativamente asociados a recurrencia en nuestro estudio.

Las limitaciones de este estudio es un enrolamiento retrospectivo, con un seguimiento prospectivo relativamente corto, pérdida de pacientes en el seguimiento, centro terciario como mayor aporte de pacientes, lo que lleva a sesgo de selección. Se necesitan estudios prospectivos con mayor número de pacientes con el evento y un seguimiento a largo plazo para mayor validez externa.

\section{Referencias}

1.- Kendig E, Dyken P, Hernández N, et al: Febrile Seizures. NIH Consens Statement Online 1980; 3: 1-10.

2.- Annegers JF, Blakely SA, Hauser WA, Kurland LT: Recurrence of febrile convulsions in a population-based cohort. Epilepsy Res 1990; 5: 209-16.

3.- Suboit T: Epidemiology of febrile and afebrile convulsions in Children in Japan. Neurology 1984; 34: $175-81$.

4.- Annegers JF, Hausser WA, Elveback LR, Kurland LT: The risk of epilepsy following febrile convulsions. Neurology 1979; 29: 297-303.

5.- Berg AT, Shinnar S: Unprovoked seizures in children with febrile seizures: short-term outcome. Neurology 1996; 47: 562-8.

6.- Practice Parameter: The neurodiagnostic evaluation of the child with a first febrile seizure. American Academy of Pediatrics. Provisional Committee on Quality Improvement, Subcommittee on febrile Seizures. Pediatrics 1996; 97: 769-72.

7.- Shinnar S, Pellock JM, Moshe SL, et al: In whom does status epilepticus occur: age -related differences in children.Epilepsia 1996; 37: 126-33.

8.- Vestergaard M, Pedersen MG, Ostergaard JR, Pedersen $C B$, Olsen J, Christensen J: Death in children with febrile seizures: a population-based cohort study. Lancet 2008; 9; 372 (9637): 457-63.

9.- Falconer MA: Mesial temporal sclerosis as a common cause of epilepsy: aetiology, treatment and prevention. Lancet 1974; ii: 767-709.

10.- Commission on Epidemiology and Prognosis: International league against Epilepsy Guidelines for epidemiology studies on epilepsy. Epilepsia 1993; 34: 592-6.

11.- Steering Committee on Quality Improvement and Management, Subcommitte on Febrile Seizures: Clinical Practice Guideline for the Long-term Management of the Child With Simple Febrile Seizures. Pediatrics 2008; 121: 1281-6.

12.- Berg AT, Shinnar S, Hauser WA, et al: A prospective study of recurrent febrile seizures. N Engl J Med 1992; 327: 1122-7.

13.- Berg AT, Shinnar S, Hauser WA, Leventhal JM: Predictors of recurrent febrile seizures: a metaanalytic 
review. J Pediatr 1990; 116: 329-37.

14.- Wolff M, Cassé-Perrot C, Dravet C: Severe Myoclonic Epilepsy of Infants (Dravet Syndrome): Natural History and Neuropsychological Findings Epilepsia 2006; 47: 45-8.

15.- Fukuyana Y, Seki T, Ohtsuka C, Miura H, Hara M:
Practical guideline for physicians in the management of febrile seizures. Brain Dev 1996; 18: 479-84.

16.- Okumura A, Ishiguro Y, Sofue A, et al: Treatment and outcome in patients with febrile convulsion associate with epileptiform discharges on electroencephalography/Brain \& Development 2004; 26: 241-4. 